\title{
Integración del control químico y etológico para la supresión poblacional de Blatella germanica (Linnaeus) (Dictyoptera: Blatellidae) en Lima, Perú
}

\author{
JOSÉ IANNACONE* y LORENA ALVARIÑO*
}

\author{
INTEGRATION OF CHEMICAL AND ETHOLOGICAL CONTROL FOR POPULATION \\ SUPPRESSION OF Blatella germanica (LINNAEUS) (DICTYOPTERA: \\ BLATELLIDAE) IN LIMA, PERU
}

\begin{abstract}
German cockroaches Blatella germanica (Linnaeus, 1767) is an arthropod specie of importance in Public Health. Effectiveness of integration chemical and ethological control for suppression of $\boldsymbol{B}$. germanica was evaluated. Experimental tests in a nursery school of Surquillo Municipality District, Lima, Peru were performed. First trial: Two formulations of cypermethrin, wet dust and emulsify concentrate were evaluated on B. germanica. 38 craft sticky traps impregnated with food bait were placed. Counts were performed at 2 days, 5 days and 8 days, through a count of small and big nymphs, and, adult males and females. Second trial: A smoking cypermethrin was evaluated. 16 craft sticky traps without natural food bait and with the same characteristics of first trial were placed. Counts were performed at 10 days, through a count of $\boldsymbol{B}$. germanica in two densities of infestation. Third trial: Susceptibility of B. germanica to three insecticides (cypermethrin 3\%o; cypermethrin 8\%o, and chlorpyrifos + cypermethrin 8\%o) under laboratory conditions with two systems of application by contact: residual (impregnate with filter paper) at 3 days, 6 days, 14 days and 21 days, and direct spray at 3 days were determined. Wet dust presented higher effectiveness at 8 days of exposure on $B$. germanica was found. Smoking cypermethrin presented effectiveness in population suppression only at low densities of $\boldsymbol{B}$. germanica. At laboratory conditions at 3 days exposure, the three insecticides showed an effectiveness of 100\% when were applied by spraying. Residual effect of $100 \%$ at 21 days only was observed with chlorpyrifos + cypermethrin. Possibilities of integration of ethological and chemical control for population supression of $\boldsymbol{B}$. germanica in Lima, Peru were analyzed.
\end{abstract}

Key words: adult, Blatella, chemical control, cockroach, ethological control, nymph, Peru.

\section{INTRODUCCIÓN}

Las cucarachas son plagas domésticas persistentes en áreas urbanas a nivel mundial ${ }^{1-3}$. De aproximadamente 4.000 cucarachas existentes, Blatella germanica (Linnaeus, 1767) "Cucaracha alemana”, es la especie sinantrópica más abundante y con una distribución cosmopolita ${ }^{4}$. Además de las molestias que ocasiona, afecta la economía y se considera de gran importancia médica pues transmite innumerables organismos patógenos como: virus, hongos, helmintos y bacterias; esta especie también es responsable de serias enfermedades alérgicas como el asma

* Laboratorio de Invertebrados. Facultad de Ciencias Biológicas. Universidad Ricardo Palma. Avenida Benavides 5440, Santiago de Surco, Lima 33, Perú. 
bronquial y la rinitis alérgica ${ }^{5-7}$. La presencia de cucarachas en ambientes urbanos, como guarderías infantiles puede causar serios problemas en los infantes ${ }^{2}$. Las cucarachas alemanas están distribuidas heterogéneamente en los diferentes ambientes que infectan y se señala que para el control de esta especie se ha usado principalmente el control químico ${ }^{8}$.

Los efectos toxicológicos de los insecticidas en la cucaracha alemana ha sido bien documentados y revisados por numerosos autores ${ }^{1,4,9-11}$. Dentro de las estrategias de control químico se incluyen aplicación de insecticidas químicos, en polvo, aerosoles y tratamientos en ultrabajo volumen con efectos blaticidas ${ }^{4,12-13}$.

Por ende, el presente estudio tuvo como objetivos integrar el control etológico y el químico para la supresión poblacional de $B$. germanica (Dictyoptera: Blatellidae) en Lima, Perú determinado por tres ensayos: 1) la susceptibilidad de $B$. germanica al insecticida piretroide (cipermetrina 1,5\%) en dos formu-laciones, polvo mojable (PM) y concentrado emulsionable (CE) empleando trampas pegantes con atrayentes alimenticios; 2) la efectividad de la cipermetrina (fumígeno) en trampas artesanales pegantes sin atrayentes naturales, y 3 ) la susceptibilidad de B. germanica a tres insecticidas (cipermetrina 3\%o; cipermetrina $8 \%$ y clorpirifos $50 \%$ + cipermetrina $8 \%$ o) bajo condiciones de laboratorio por contacto residual (Impregnación en papel filtro) y por aspersión directa.

\section{MATERIAL Y MÉTODOS}

\section{Área de estudio y fuente de organismos prueba}

Ensayo 1 y 2: Las pruebas experimentales se realizaron en una Cuna Guardería Infantil "Tito", perteneciente a la Municipalidad Distrital de Surquillo, Lima, Perú. La cual presenta problemáticas por infestación de $B$. germanica por más de cinco años. El ensayo 1 se realizó de agosto a septiembre del 2001. En cambio el ensayo 2 se llevó a cabo entre enero y febrero del 2003.

Blatella germanica en una visita previa, se identificó a nivel del estado ninfal y adulto empleando las características morfológicas indicadas por Hartwood \& James ${ }^{5}$, depositándose material tipo en el Museo de Entomología del
Programa Nacional de Control Biológico del Servicio Nacional de Sanidad Agraria, Ministerio de Agricultura (PNCB, SENASA, MIAGRI).

Ensayo 3: Se recolectaron numerosos individuos adultos $(n=200)$, ninfales $(n=250)$ y ootecas $(n=25)$, procedentes del Albergue Municipal "Rosario Araoz Pinto", San Juan de Miraflores, Lima, Perú. La colecta se realizó en recipientes de plástico transparentes de $500 \mathrm{~mL}$ y de $1.000 \mathrm{~mL}$ con la ayuda de una espátula de metal, y se trasladó al laboratorio de Invertebrados de la Facultad de Ciencias Biológicas de la Universidad Ricardo Palma (URP). Los adultos, ninfas y ootecas de $B$. germanica fueron introducidos a envases de plástico transparentes de $1.000 \mathrm{~mL}$ de capacidad. Internamente se colocaron por cada envase plástico: un papel filtro Whatman ${ }^{\circledR} \mathrm{N}^{\circ} 1$ en el fondo del recipiente, dos trozos de esponja de $1 \mathrm{~cm}^{3}$ previamente embebido con agua desionizada ad libitum y dos trozos enteros de galleta de soda con un área de $1 \mathrm{~cm}^{2}$ (Soda Field® (Kraft Foods Perú, S.A., Lima, Perú)). En cada envase fueron colocados separadamente adultos, ninfas y ootecas. El ambiente de cría presentó una temperatura de 23 $\pm 2^{\circ} \mathrm{C}$ y con fotoperíodo $(12 \mathrm{~h}$ luz: $12 \mathrm{~h}$ oscuridad). Los adultos fueron aclimatados previamente por una semana antes de la realización de los bioensayos de susceptibilidad.

\section{Insecticidas}

Ensayo 1: Los plaguicidas empleados como tratamientos fueron dos: alfa $(+)$ cipermetrina (dosis $=1,5 \%$, formulación polvo mojable $)$; alfa (+) cipermetrina $($ dosis $=1,5 \%$, formulación concentrado emulsionable) y testigo (agua de grifo declorinada). Los tres tratamientos se realizaron en una sola aplicación al caer el sol. Los productos fueron aplicados con un pulverizador Jacto ${ }^{\circledR}$ (HD 300 de 10 L, Santiago de Chile, Chile) y se llevó a cabo con una boquilla de Abanico Plano, 80 - 02, con una cobertura estandarizada de $8 \mathrm{~L}=150 \mathrm{~m}^{-2}$.

Ensayo 2: Se usó la cipermetrina en formulación tipo pastilla fumígena. La composición química es $1,3 \%$ de cipermetrina y $98,7 \%$ de inertes. Se empleó una pastilla por cada $20 \mathrm{~m}^{2}$ de habitación, la cual fue colocada sobre el suelo en un trozo de mayólica de $20 \mathrm{~cm}$ x $20 \mathrm{~cm}$.

Ensayo 3: Los plaguicidas empleados como tratamientos fueron: cipermetrina $($ dosis $=3 \%$ ), cipermetrina $($ dosis $=8 \%$ ) (Stockade $\AA)$ y 
clorpirifos $50 \%$ + cipermetrina (dosis $=8 \%$ o). Para la preparación de las soluciones empleadas en los ensayos de susceptibilidad para evaluar supervivencia de $B$. germanica se disolvieron las sustancias químicas en agua desionizada $(\mathrm{pH}$ $=7,12 ;$ conductividad específica $=70$ umhos $\left.\cdot \mathrm{cm}^{-1}\right)^{14}$.

\section{Pruebas experimentales}

Ensayo 1: Los experimentos se iniciaron con la colocación de 35 trampas artesanales con atrayentes para $B$. germanica en los ambientes de la Cuna Guardería Infantil de Surquillo, Lima, Perú. Cada trampa fue confeccionada empleando una base de cartulina Canson ${ }^{\circledR}$ de $15 \mathrm{~cm}$ de diámetro y un cono truncado de material flexible poroso como tapa del mismo diámetro de la base de cartulina y con 7,5 cm de diámetro en la base menor superior. Además, se realizaron cuatro aperturas de $2 \mathrm{~cm}$ de altura y $5 \mathrm{~cm}$ de ancho. Sobre las bases de cartulina Canson, internamente se colocaron papeles filtro Whatman ${ }^{\circledR} \mathrm{N}^{\circ} 1 \mathrm{de}$ $15 \mathrm{~cm}$ de diámetro sobre los que previamente se pulverizó una mezcla asperjada a un $1,5 \mathrm{~mL}$ de suspensión por papel formada por ácido bórico $(50 \mathrm{~g})$, mantequilla de maní (50 g) y cerveza comercial $(300 \mathrm{~mL})$, en una proporción de 1:1:6, respectivamente, la cual fue vigorosamente agitada, previa a su aplicación sobre el papel filtro $^{2}$. Además, se le adicionó un pegamento comercial denominado Temocid® (Kollant, Italia) sobre cada uno de los papeles filtro ${ }^{15}$. Adicionalmente, fueron colocadas tres trampas más, $48 \mathrm{~h}$ después, principalmente en las zonas de mayor infestación por B. germanica. Teniendo la siguiente distribución (Figura 1): un total de 15 trampas en la cocina, 4 trampas en el baño $\left(2^{\text {do }}\right.$ Piso), 2 trampas en cuarto pequeño junto al baño $\mathrm{y}$ una en un cuarto grande ( $2^{\mathrm{do}}$ piso). Seis en un cuarto grande junto a la cocina, 3 en un cuarto pequeño junto a la cocina, tres en el almacén de alimentos, una en cada una de las cuatro aulas de la guardería. En cada período de lectura las trampas fueron cambiadas por nuevas. La colocación de las 35 trampas en cada uno de los ambientes seleccionados fueron realizados $48 \mathrm{~h}$ (2 d) previos a la aplicación de los tratamientos. Adicionalmente, fueron colocadas tres trampas más el día de la pulverización de los tratamientos, principalmente en las zonas de mayor infestación por B. germanica. La cipermetrina $1,5 \%$ en polvo mojable se aplicó en la cocina, y en los cuartos grandes y pequeños adjuntos a la cocina. La cipermetrina $1,5 \%$ en concentrado emulsionable se aplicó en el baño, cuarto pequeño y grande del segundo piso; así como en los cuarto de almacén de alimento. El control o testigo estuvo conformado por las cuatro aulas de la guardería (Figura 1). Se colocó 24 trampas en las áreas asperjadas con polvo mojable, 10 en las pulverizadas con concentrado emulsionable y 4 con agua de grifo (testigo). La efectividad de los tratamientos fue evaluada a las $48 \mathrm{~h}$ (2d), 120 h (5d) y 192 h (8d), mediante el conteo de los diferentes estadios de B. germanica: ninfas pequeñas $(<3 \mathrm{~mm})$, ninfas grandes $(>3 \mathrm{~mm})$, machos y hembras adultos en las trampas pegantes.

Ensayo 2: Los experimentos se iniciaron con la colocación de 3 trampas artesanales sin atrayentes alimenticios para $B$. germanica en tres diferentes ambientes con bajas densidades la cucaracha alemana: baño $\left(7 \mathrm{~m}^{2}\right)$ y cuarto pequeño $\left(18 \mathrm{~m}^{2}\right)$ del $2^{\text {do }}$ piso y cuarto grande del $2^{\text {do }}$ piso $\left(80 \mathrm{~m}^{2}\right)$ de la Cuna Guardería Infantil de Surquillo, Lima, Perú (Figura 1). En cambio a altas densidades de la cucaracha alemana las 13 trampas fueron colocadas en la cocina (Figura 1). Las trampas fueron similares a las usadas en el ensayo 1. Además, se le adicionó un pegamento comercial denominado Temocid® sobre cada una de las cartulinas ${ }^{15}$. Las trampas fueron expuestas por 10 días antes de la aplicación del producto fumígeno y fueron cambiadas por trampas nuevas posteriormente a la aplicación. La efectividad de las trampas artesanales pegantes fue evaluada a los $10 \mathrm{~d}$ mediante el conteo de los diferentes estadios de $B$. germanica: ninfas pequeñas $(<0=3 \mathrm{~mm})$, ninfas grandes $(>3$ $\mathrm{mm}$ ), machos y hembras adultas.

Ensayo 3: Las pruebas de susceptibilidad se iniciaron con ninfas y adultos (hembras y machos). Se realizaron bioensayos estáticos utilizando un total de 5 individuos envase ${ }^{-1}$, con cuatro repeticiones: adultos hembras; adultos machos y ninfas. Los envases de experimentación utilizados fueron placas de petri de plástico descartables de 100 x $15 \mathrm{~mm}$ usados como "arenas" con papel Whatman ${ }^{\circledR} \mathrm{n}^{\circ} 1$ (Inc. 200 Park Avenue, Suite 210. Florham Park, NJ 07932 USA). Los tres insecticidas empleados, la cipermetrina a dos dosis y la mezcla de clorpirifos y cipermetrina fueron aplicados por aspersión o por efecto residual. Para los ensayos directos de 
aspersión, se colocaron sobre una de las placas de petri una malla de $0,5 \mathrm{~mm}$ de diámetro, la cual fue asperjada con 1,5 mL de suspensión del insecticida para cada una de las concentraciones y para el control. En cambio, para los ensayos de contacto por efecto residual, se realizaron asperjando directamente sobre el papel filtro, previo secado a temperatura de laboratorio de 23 $\pm 2{ }^{\circ} \mathrm{C}$. Además, a cada envase experimental, se colocó trozos de esponja de $0,25 \mathrm{~cm}^{3}$ previamente embebido con agua desionizada y un trozo entero de galleta de un $1 \mathrm{~cm}^{2}$ (Soda Field $\left.{ }^{\circledR}\right)$. La duración de la prueba para los ensayos de aspersión fue $3 \mathrm{~d}$ y para los de efecto residual fue de $3 \mathrm{~d}, 6 \mathrm{~d}$, $14 d$ y $21 d$ de exposición. El indicador de la prueba de mortalidad fue la mortalidad de los especímenes, durante $15 \mathrm{~s}$ de observación bajo la acción del microscopio estereoscopio. El tratamiento usado como testigo fue a base de agua destilada. El protocolo de bioensayo se realizó empleando el recomendado por la Organización Mundial de la Salud (OMS) ${ }^{16}$.

\section{Diseño experimental y tratamiento estadístico}

Ensayo 1: Se empleó la prueba de t de student para comparar la densidad de cucarachas antes y después de la aplicación de la cipermetrina previa transformación de los datos a $\log \mathrm{X}+1$. Se compararon los números totales y de cada uno de los estadios desarrollo de B. germanica, para las trampas artesanales pegantes en los tres periodos de lectura ( $48 \mathrm{~h}, 120 \mathrm{~h}$ y $192 \mathrm{~h}$ ) mediante la prueba de ANDEVA, previa transformación de los datos a $\log \mathrm{X}+1$, en $\operatorname{los}$ casos de existir diferencias significativas se utilizó la prueba de Tukey ${ }^{17}$. Además se usó el estadístico G para evaluar si existían diferencias en las frecuencias relativas para cada uno de los estados de desarrollo de $B$. germanica.

Ensayo 2: Se compararon los números totales de los estadios de desarrollo de $B$. germanica, antes y después de la aplicación de las pastillas fumígenas mediante la prueba de ANDEVA, previa transformación de los datos a arcoseno. En los casos de existir diferencias significativas se utilizó la prueba de Tukey ${ }^{17}$.

Ensayo 3: Las pruebas de toxicidad aguda de contacto por aspersión y residual sobre $B$. germanica, para los tres insecticidas se evaluaron con cuatro repeticiones, más un control, en un diseño experimental en bloque completamente randomizado (DBCR): 4 x 4 . La eficacia de los tratamientos se evaluó a través de un ANDEVA, previa transformación de los datos a raíz cuadrada del arcoseno. En el caso de existir diferencias significativas entre las réplicas y los tratamientos se realizó la prueba de Tukey ${ }^{17}$. Cuando las mortalidades fueron mayores del $10 \%$ en el control, se utilizó la fórmula de Abbott, para la corrección de los porcentajes de mortalidad en los tratamientos evaluados.

\section{RESULTADOS}

Ensayo 1: La Tabla 1 nos indica que el polvo mojable de cipermetrina presento diferencias significativas en el número total de $B$. germanica entre los cinco y ocho días de exposición; con una reducción del $76,5 \%$ del número total de $B$. germanica entre ambos periodos de exposición. La reducción del número de cucarachas fue de $54,67 \%$ en el tratamiento con Concentrado emulsionable. Los ambientes asperjados con el concentrado emulsionable presentaron el mayor número de cucarachas por trampa asociado a la terma del baño (trampas 14 y 16) (Figura 1). Además material biológico de $B$. germanica recogidos por el proceso de barrido de los ambientes, detectó en la cocina: 4 especímenes (1 ninfa grande y 3 ninfas pequeñas) y en el baño 12 especímenes (4 ninfas grandes, 7 ninfas pequeñas y 1 macho). En la cocina el sitio con mayor concentración de cucarachas alemanas fue la refrigeradora (Trampas 1, 2, 10 y 12).

Se observó la siguiente secuencia en orden descendente de $B$. germanica: el número de ninfas pequeñas $(5,1 \pm 17,8, \mathrm{n}=114,0-126)>$ ninfas grandes $(0,9 \pm 2,8, \mathrm{n}=114,0-15)>$ adultos machos $(0,5 \pm 1,6, \mathrm{n}=114,0-9)>$

Tabla 1. Efectividad de las dos formulaciones de cipermetrina sobre $B$. germanica en la Cuna Guardería Infantil de Surquillo, Lima, Perú

\begin{tabular}{|c|c|c|c|c|}
\hline \multirow[b]{2}{*}{ Formulaciones } & \multicolumn{4}{|c|}{ No total de $B$. germanica trampa ${ }^{-1}$} \\
\hline & $\begin{array}{c}120 \mathrm{~h} \\
(5 \mathrm{~d})\end{array}$ & $\begin{array}{c}192 \mathrm{~h} \\
(\mathbf{8 d})\end{array}$ & $\begin{array}{c}\text { t de } \\
\text { Student }\end{array}$ & $\mathbf{P}$ \\
\hline Polvo mojable & 43,3 & 10,1 & 2,11 & 0,04 \\
\hline $\begin{array}{l}\text { Concentrado } \\
\text { emulsionable }\end{array}$ & 5,1 & 2,2 & 0,32 & 0,75 \\
\hline Testigo o blanco & 0,0 & 0,5 & 1,00 & 0,39 \\
\hline
\end{tabular}

$\mathrm{P}=$ Probabilidad 


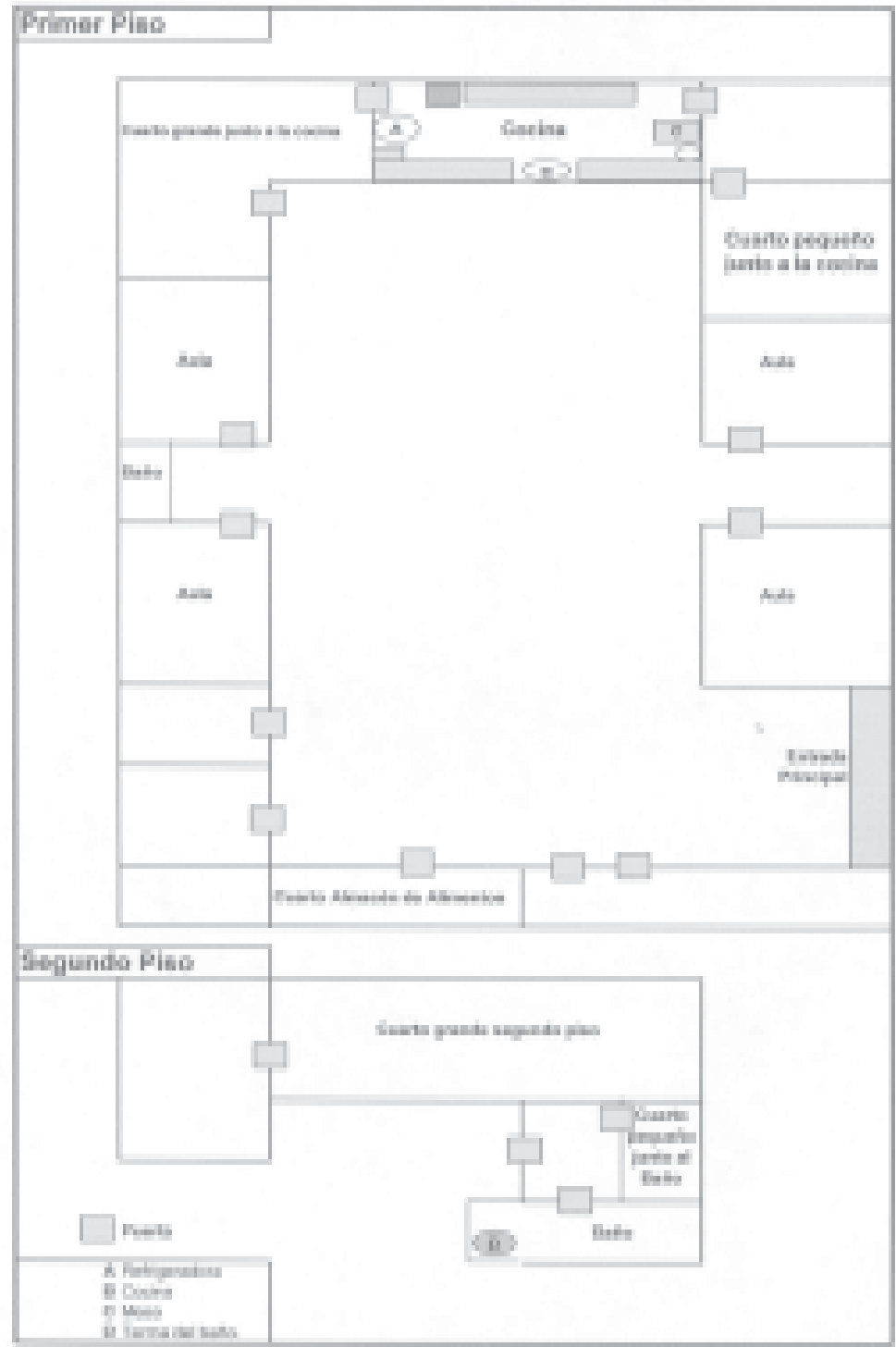

Figura 1. Diagrama de los ambientes de la cuna guardería infantil, Municipalidad Distrital de Surquillo, utilizados para los ensayos experimentales de campo para el control de B. germanica. hembras $(0,2 \pm 1,0, \mathrm{n}=114,0-7)$ en cada una de las trampas con atrayentes alimenticios. Además, se observó la siguiente secuencia porcentual en orden descendente para los cuatro estadios de desarrollo: ninfas pequeñas $(74,1 \%$, total $=581)$ $>$ ninfas grandes $(14,1 \%$, total $=111)>$ adultos machos $(7,9 \%$, total $=62)>$ adultos hembra $(3,8 \%$, total $=30)$. La Tabla 2 , muestra que según la prueba de ANDEVA no existieron diferencias significativas entre los tiempos de exposición de las trampas: $2 \mathrm{~d}, 5 \mathrm{~d}$ y $8 \mathrm{~d}$, entre las ninfas pequeñas, ninfas grandes y hembras adultas. Sin embargo, si se observaron diferencias según la prueba de Tukey entre los machos adultos: $5 \mathrm{~d}>2 \mathrm{~d}=8 \mathrm{~d}$ (Tabla 2). La prueba $\mathrm{G}$, mostró que no existieron diferencias para cada uno de los estados desarrollo con relación a las frecuencias relativas (número de trampas presentes con cada de los estados de desarrollo) (Tabla 2).

Ensayo 2: Se observó la siguiente secuencia en orden descendente de B. germanica a bajas densidades por trampa antes del empleo de las pastillas fumígenas con cipermetrina: el número de ninfas pequeñas $>$ ninfas grandes $>$ adultos machos $>$ hembras en cada una de las trampas 
Tabla 2. Análisis comparativo del número de ninfas pequeñas, ninfas grandes, machos y hembras adultas de $B$. germanica entre los tres tiempos de colecta en trampas pegantes con atrayentes alimenticios

\begin{tabular}{lcccrr}
\hline Tiempo & Ninfas pequeñas & Ninfas grandes & machos & hembras & Totales \\
\hline 2d $(\mathrm{n}=38)$ & $2,9 \mathrm{a}$ & $0,8^{\mathrm{a}}$ & $1,1 \mathrm{~b}$ & $0,1 \mathrm{a}$ & $4,1 \mathrm{a}$ \\
$5 \mathrm{~d}(\mathrm{n}=38)$ & $10,0 \mathrm{a}$ & $1,7^{\mathrm{a}}$ & $0,2 \mathrm{a}$ & $0,5 \mathrm{a}$ & $13,3 \mathrm{a}$ \\
8d (n=38) & $2,3 \mathrm{a}$ & $0,3^{\mathrm{a}}$ & $0,2 \mathrm{a}$ & $0,05 \mathrm{a}$ & $3,0 \mathrm{a}$ \\
Estadístico F & 2,2 & 2,1 & 4,03 & 2,91 & 2,4 \\
P & 0,11 & 0,11 & 0,02 & 0,06 & 0,09 \\
$\%$ & 74,1 & 14,1 & 7,9 & 3,8 & 100
\end{tabular}

$\mathrm{P}=$ Probabilidad $; \mathrm{F}=$ Estadístico Fisher; $\%=$ Porcentaje. Letras minúsculas en una línea vertical señalan que los promedios son estadísticamente iguales.

Tabla 3. Análisis comparativo del número de trampas con presencia de ninfas pequeñas, ninfas grandes, machos y hembras adultas de $B$. germanica entre los tres tiempos de colecta en trampas pegantes con atrayentes alimenticios

\begin{tabular}{lccrrr}
\hline Tiempo & Ninfas pequeñas & Ninfas grandes & machos & hembras & Totales \\
\hline 2d $(\mathrm{n}=38)$ & 15,7 & 17,7 & 13,1 & 7,8 & 15,7 \\
5d $(\mathrm{n}=38)$ & 28,9 & 23,6 & 21,1 & 18,4 & 31,5 \\
8d $(\mathrm{n}=38)$ & 31,5 & 18,4 & 7,8 & 2,6 & 31,5 \\
Promedio & $25,43 \%$ & $19,29 \%$ & $14,03 \%$ & $9,64 \%$ & $26,31 \%$ \\
Estadístico G & 2,82 & 2,35 & 2,45 & 1,15 & 1,94 \\
P & $>0,05$ & $>0,05$ & $>0,05$ & $>0,05$ & $>0,05$ \\
\hline
\end{tabular}

$\mathrm{P}=$ Probabilidad .

Tabla 4. Análisis comparativo del número de ninfas pequeñas, ninfas grandes, machos y hembras adultas de $B$. germanica a bajas densidades expuestas a la aplicación de la pastilla fumígena de cipermetrina en las trampas pegantes sin atrayentes naturales

\begin{tabular}{lccccr}
\hline ExposiciónNinfas pequeñas & Ninfas grandes & machos & hembras & Totales \\
\hline 10 d antes & 2,67 & 2,33 & 1 & 0,33 & 6,33 \\
10 d después & 0 & 0 & 0,67 & 0 & 0,67 \\
\% de Efectividad & 100 & 100 & 33 & 100 & 89,41 \\
\hline
\end{tabular}

sin atrayentes alimenticios (Tabla 4). Además, se observó la siguiente secuencia porcentual en orden descendente para los cuatro estadios de desarrollo antes de la aplicación de las pastillas fumígenas: ninfas pequeñas $(42,18 \%)>$ ninfas grandes $(36,8 \%)>$ adultos machos $(15,8 \%)>$ adultos hembra $(5,22 \%)$. La efectividad para ninfas pequeñas, Ninfas grandes y hembras fue del $100 \%$ : En cambio para los machos fue sólo de $33 \%$ (Tabla 4). La efectividad global fue de $89,41 \%$.

Se encontró la siguiente secuencia en orden descendente de $B$. germanica a altas densidades por trampa antes del empleo de las pastillas fumígenas con cipermetrina: el número de ninfas pequeñas $>$ ninfas grandes $>$ adultos machos $>$ hembras en cada una de las trampas sin atrayentes alimenticios (Tabla 5). Además, se observó la siguiente secuencia porcentual en orden descendente para los cuatro estadios de desarrollo antes de la aplicación de las pastillas fumígenas: ninfas pequeñas $(55,71 \%)>$ ninfas grandes $(19,79 \%)>$ adultos machos $(15,66 \%)>$ adultos hembra $(8,84 \%)$. La efectividad para ninfas pequeñas y ninfas grandes fue del $0 \%$. En cambio para los machos fue de $36,76 \%$ y para las hembras fue de $17,1 \%$ (Tabla 5). La efectividad global fue de $0,36 \%$. En altas densidades de infestación por B. germanica, la pastilla fumígena 
Tabla 5. Análisis comparativo del número de ninfas pequeñas, ninfas grandes, machos y hembras adultas de $B$. germanica a altas densidades expuestas a la aplicación de la pastilla fumígena de cipermetrina en las trampas pegantes sin atrayentes naturales

\begin{tabular}{lccccr}
\hline Exposición & Ninfas pequeñas & Ninfas grandes & Machos & hembras & Totales \\
\hline 10 d antes & 44,62 & 15,85 & 12,54 & 7,08 & 80,09 \\
10 d después & 47,07 & 18,93 & 7,93 & 5,87 & 79,8 \\
\% de Efectividad & 0 & 0 & 36,76 & 17,1 & 0,36 \\
\hline
\end{tabular}

Tabla 6. Efecto de tres insecticidas en la mortalidad de B. germanica por aspersión y por efecto residual

\begin{tabular}{lccrrr}
\hline Tratamientos & $\mathbf{3 d}^{\mathbf{1}}$ & $\mathbf{3 d}^{\mathbf{2}}$ & $\mathbf{6 d}^{\mathbf{2}}$ & $\mathbf{1 4 d}^{\mathbf{2}}$ & $\mathbf{2 1 d}^{\mathbf{2}}$ \\
\hline Testigo & $15 \mathrm{a}$ & $0 * \mathrm{a}$ & $0 \mathrm{a}$ & $0 \mathrm{a}$ & $0 \mathrm{a}$ \\
Cipermetrina (3\%o) & $100 \mathrm{~b}$ & $8 \mathrm{ab}$ & $16 \mathrm{~b}$ & $16 \mathrm{~b}$ & $25 \mathrm{~b}$ \\
Cipermetrina (8\%) & $100 \mathrm{~b}$ & $16 \mathrm{~b}$ & $16 \mathrm{~b}$ & $25 \mathrm{~b}$ & $35 \mathrm{~b}$ \\
Clorpirifos + Cipermetrina 8\%o) & $100 \mathrm{~b}$ & $100 \mathrm{c}$ & $100 \mathrm{c}$ & $100 \mathrm{c}$ & $100 \mathrm{c}$ \\
\hline
\end{tabular}

Letras diferentes indican efectos de mortalidad estadisticamente significativos. 1 = aspersión; 2 = efecto residual.

* = Testigo ajustado empleando la fórmula de Abbott.

de cipermetrina no es efectiva contra los estadios ninfales.

Ensayo 3: La Tabla 6 muestra los porcentajes de mortalidad obtenidos a $3 \mathrm{~d}$ de exposición en bioensayos de aspersión sobre $B$. germanica. Se observó para todos los casos una mortalidad del $100 \%$ sobre adultos hembras, machos y ninfas para los tres insecticidas: cipermetrina 3\%o, cipermetrina $8 \%$ y clorpirifos + cipermetrina $6 \%$. En relación a los porcentajes de mortalidad en el bioensayo de efecto residual, a 3d, 6d, 14d y 21d de exposición se observó el siguiente orden de toxicidad decreciente: clorpirifos + cipermetrina $>$ cipermetrina $=$ alfacipermetrina . No se observaron diferencias en la susceptibilidad a los insecticidas entre los estados de desarrollo de $B$. germanica en los ensayos por contacto de aspersión y residual, por lo que se procedió a juntar todos los resultados. En lo bioensayos de contacto de tipo residual, las cipermetrinas mostraron a las dosis ensayadas una mortalidad no mayor del $25 \%$. La mezcla de clorpirifos + cipermetrina presentó notablemente un mayor efecto residual, inclusive luego de $21 \mathrm{~d}$ de aspersión, se colocaron nuevos individuos produciéndose un $100 \%$ de mortalidad a $3 \mathrm{~d}$ de exposición (Observación personal).

\section{DISCUSIÓN}

A pesar que diversas actividades intensivas de control contra la cucaracha alemana, tanto por las entidades gubernamentales como por la población afectada están siendo implementadas². Sin embargo, B. germanica sigue siendo una plaga de importancia en salud pública y médica, y continúa infestando edificaciones como casas y restaurantes a altas y baja densidades poblacionales ${ }^{18}$.

Recientemente, la aplicación de cebos tóxicos ha sido ampliamente empleados para el control de cucarachas. Se ha evaluado el efecto de control de varios cebos tóxicos (hidrametilon, clorpirifos, fenitrotion y imidacloprid) contra la cucaracha alemana ${ }^{19}$. El Fiproponil es un insecticida fenilpirazolido relativamente nuevo, el cual está disponible desde 1999 en formulaciones de cebo y usadas para el control de las cucarachas domésticas y de hormigas ${ }^{18}$. En el primer ensayo del presente trabajo, se empleó un atrayente alimenticio principalmente con productos naturales como mantequilla de maní y cerveza, pero con ausencia de algún producto químico sintético tóxico.

Muchos insecticidas convencionales no son 
efectivos en controlar infestaciones masivas de B. germanica, debido a su ciclo biológico corto de tres a seis meses dependiendo de la temperatura. Por ende es muy dificultoso de controlar esta especie en comparación con otras especies de cucarachas ${ }^{18}$. En el presente estudio se ha encontrado que la cipermetrina fumígena a altas densidades ha demostrado no ser eficaz en controlar a B. germanica (Tabla 5).

Por otro lado, se ha encontrado en la literatura que $B$. germanica muestra resistencia a insecticidas como el malation y el propoxur ${ }^{20-21}$. En adición, esta especie muestra una ligera tolerancia al sumition y al tetrametorin ${ }^{18}$. Gliniewickz ${ }^{16}$ ha encontrado que variedades de campo de $B$. germanica muestran una alta a moderada resistencia a la cipermetrina. Nuestros resultados muestran una mayor eficacia a los $8 \mathrm{~d}$ de exposición para el polvo mojable de cipermetrina (Tabla 1).

Estudios previos han revelado que $B$. germanica infesta masivamente con fines de cría y alimentación, la cocina de hospitales, apartamentos, hoteles, casas privadas y restaurantes, pudiendo visitar otros lugares para encontrar su alimento ${ }^{18,22}$. Estos resultados fueron similares a los nuestros, pues se encontró mayores densidades de $B$. germanica en la cocina $\mathrm{y}$ en los baños en los ensayos 1 y 2 .

Se han empleado diferentes métodos para el censo de cucarachas como el conteo visual y las trampas pegantes. El conteo visual es realizado después de las 22:30 h, por lo que se apagan las luces a las 21:30 por una h. Luego se enciende la luz y se observa y cuenta las cucarachas que se encuentran movilizándose sobre las mesas y las áreas de alimentación por $5 \mathrm{~min}$. Este método permite dividir en tres categorías de infestación a las cucarachas: alta ( $>75)$, media (25-75) y baja $(<25)$ densidad de cucarachas ${ }^{18}$. Nosotros en cambio, al igual que Tilak ${ }^{13}$ proponemos para censar cucarachas, las trampas pegantes con atrayentes alimenticios en cantidades suficientes a los lugares cercanos a sus refugios para estimar la densidad y el tipo de cucaracha presente.

Finalmente, nuestros resultados en los tres ensayos indican que el empleo de la estrategia integral de insecticidas, principalmente a base de cipermetrina con el control etológico a base de trampas pegantes con atrayente alimenticio es la recomendada para la supresión poblacional de $B$. germanica ${ }^{21}$.

\section{RESUMEN}

La cucaracha alemana Blatella germanica (Linnaeus, 1767) es una especie de artrópodo de importancia en Salud Pública. Por lo que se evaluó la efectividad de integración del control etológico y químico para la supresión de $B$. germanica. Las pruebas experimentales se realizaron en una Cuna Guardería Infantil de la Municipalidad Distrital de Surquillo, Lima, Perú. Primer ensayo: Dos formulaciones de cipermetrina en polvo mojable y concentrado emulsionable fueron evaluadas sobre $B$. germanica. Se colocaron 38 trampas artesanales pegantes impregnadas con atrayente alimenticio. Las lecturas se realizaron a los $2 \mathrm{~d}, 5 \mathrm{~d}$ y $8 \mathrm{~d}$, mediante un conteo de ninfas pequeñas y grandes, y, machos y hembras adultas. Segundo ensayo: Una cipermetrina fumígena fue evaluada. Se colocaron 16 trampas artesanales pegantes sin atrayentes naturales y con las mismas características del ensayo 1. Las lecturas se realizaron a las $600 \mathrm{~h}$, mediante el conteo de $B$. germanica en dos densidades de infestación. Tercer ensayo: Se determinó la susceptibilidad de B. germanica a tres insecticidas (cipermetrina $3 \%$; cipermetrina $8 \%$, y clorpirifos + cipermetrina $8 \%$ ) bajo condiciones de laboratorio con dos sistemas de aplicación por contacto: residual (Impregnación en papel filtro) a 3d, 6d, $14 \mathrm{~d}$ y $21 \mathrm{~d}$ y por aspersión directa a $3 \mathrm{~d}$. Se encontró que la formulación polvo mojable presentó mayor efectividad a 8d de exposición sobre $B$. germanica. El fumígeno de cipermetrina presentó efectividad en la supresión poblacional solo a baja densidad de B. germanica. En condiciones de laboratorio a $3 \mathrm{~d}$ de exposición los tres insecticidas presentaron una efectividad del $100 \%$ al ser aplicados por aspersión. El efecto residual de $100 \%$ a 21d sólo se observó con clorpirifos + cipermetrina. Se analiza la posibilidad de integración del control etológico y químico para la supresión poblacional de B. germanica en Lima, Perú.

Palabras claves: adulto, Blatella, control etológico, control químico, cucaracha, ninfa, Perú.

\section{REFERENCIAS}

1.- KRAMER RD, KOEHLER PG, PATTERSON RS, 
SLANSKY F. Effecf of sublethal propoxur exposure on male German Cockroaches (Orthoptera: Batlellidae) and their feeding behavior. J Econ Entomol 1989; 82: 842-6.

2.- SCHAL C, HAMILTON L. Integrated suppression of synanthropic cockroaches. Annu Rev Entomol 1990; 35: 521-51.

3.- FISCHER AO, MATLOVA L, DVORSKA L, et al., Nymphs of the Oriental cockroach (Blatta orientalis) as passive vectors of causal agents of avian tuberculosis and paratuberculosis. Medical Vet Entomol 2003; 17: $145-50$.

4.- APPEL A G. Laboratory and field performance of consumer bait products fro German Cockroach (Dictyoptera : Blatellidae) control. J Econ Entomol 1990; 83: 153-9.

5.- HARTWOOD R F, JAMES M T. Entomology in human and animal health. $7^{\text {th }}$ Ed. Macmillan Publ, New York. pp. 102-110. 1979.

6.- IANNACONE J, VELÁSQUEZ K, ARRASCUE A. Fauna Parasitaria de Periplaneta americana Linnaeus en un distrito de Lima. Rev Peru Biol 1999; 6: 54-60.

7.- DÍAZ C, PÉREZ M, MAGDALENA R M, et al. Resistencia a insecticidas en cepas de terreno de la especie Blattella germanica procedentes de Santiago de Cuba. Rev Cubana Med Trop 200; 52: 24-30.

8.- SODERLUND D M, BLOOMQUIST J R. Neurotoxic actions of pyrethroid insecticides. Ann Rev Entomol 1989; 34: 77-96.

9.- SCHNEIDER B M, BENNETT G W. Comparative studies of several methods for determining the repellency of blatticides. J Econ Entomol 1985; 78: 874-8.

10.- DURIER V, RIVAULT C. Secondary transmission of toxic baits in German Cockroach (Dictyoptera: Blatellidae). J Econ Entomol 2000a; 93: 434-40.

11.- DURIER V, RIVAULT C. Comparison of toxic baits for controlling the cockroach, Blatella germanica: attractiveness and feeding stimulation. Med Vet Entomol 2000b; 14: 410-8.

12.- BARCAY S J, SCHNEIDER B M, BENNETT G W.
Influence of insecticide treatment on german cockroach (Dictyoptera: Blatellidae) movement and dispersal within apartments. J Econ Entomol 1990; 83: 142-7.

13.- TILAK R, AGRAWAL V K, DUTTA J. Field performance of cyphenothrin: an integrated insecticide strategy against German cockroaches (Dictyoptera: Blatellidae). J Vect Borne Dis 2005; 42: 68-73.

14.- IANNACONE J, ALVARIÑO L, MANSILLA J. Actividad insecticida de cuatro extractos botánicos sobre larvas de los mosquitos Culex quinquefasciatus (Díptera: Culicidae) y Chironomus calligraphus (Díptera: Chironomidae). Wiñay Yachay (Perú) 2002; 6: 56-71.

15.- LEE D K. 2000. Relative density and annual abundance of domestic cockroaches in restaurants in Pusan. Korea. Korean J Entomol 2000; 30: 187-92.

16.- GLINIEWICKZ A, KRZEMINSKA A, SAWICKA B. Susceptibility of cockroaches Blatella germanica L. collected from hospitals to selected pyrethroid and carbamate insecticides. Rocz Panstw Zakl Hig 1996; 47: 333-41.

17.- ZAR J H. Biostatistical Analysis. $3^{\text {er }}$ Ed. Prentice-Hall, Inc. Upper Saddle River. New Yersey. 662 pp. 1996.

18.- REE H I, LEE I Y, JEON S H, YONG T S. Field trial on the control effect of fipronil bait against German cockroaches. Korean Jour Parasitol 2006; 44: 255-7.

19.- AGRAWAL V K, TILAK R. Field performance of imidacloprid gel bait against German cockroaches (Dictyoptera: Blatellidae). Indian J Med Res 2006; 124: 89-94.

20.- BRET B L, ROSS M H. Behavioral responses of the German cockroach, Blatella germanica (L.) (Orthoptera: Blatellidae), to a propoxur formulation. J Econ Entomol 1986; 79: 426-30.

21.- AGRAWAL V K, TILAK R, DUTTA GUPTA K K. Efficacy of synthetic pyrethroid and propoxur aerosol in the control of German cockroaches (Dictyoptera: Blatellidae) in cookhouses. J Vect Borne Dis 2005; 42: 117-21.

22.- KUTRUP B. Cockroach Infestation in Some Hospitals in Trabzon, Turkey. Turk J Zool 2003; 27: 73-7. 